\title{
Delay-dependent Stability of Uncertain Distributed Time-delay Systems
}

\author{
Cheng Wang ${ }^{1, a}$, Qing Zhang ${ }^{1, b}$ and Jianping Gan ${ }^{2, c}$ \\ ${ }^{1}$ College of Mathematics and Computer Science, Huanggang Normal University, \\ Huanggang 438000, China \\ ${ }^{2}$ Institute of Demographic Ecology and Resources Management, \\ Huanggang Normal University, Huanggang, 438000, China \\ a wangc80@163.com, ${ }^{\mathrm{b}}$ zhangqing@hgnu.edu.cn, ${ }^{\mathrm{c}}$.jpgan@hgnu.edu.cn
}

Keywords: Delay-dependent stability, time-delay, linear matrix inequality (LMI)

\begin{abstract}
In this paper, the problem of stability analysis of uncertain distributed time-delay systems is investigated. Systems with norm-bounded parameter uncertainties are considered. By taking suitable Lyapunov-Krasovskii functional and free weighting matrices, a delay-dependent sufficient condition is derived in terms of linear matrix inequality (LMI). The condition obtained in this paper can be tested numerically very efficiently using interior point algorithms.
\end{abstract}

\section{Introduction}

Over the past decades, time-delay systems have been extensively studied because of their important applications. It is well known that time-delay frequently occurs and is a major source of instability and poor performance. Therefore, much effort has been invested in the analysis of time-delay systems [1-5]. As is well known now, stability is one of the main properties of uncertain distributed time-delay systems. On the other hand, it has been recognized that the time delay often occurs in distributed systems, and may cause oscillation and instability. Thus, the problem of stability analysis for uncertain distributed time-delay systems has become a topic of great theoretic and practical importance in the literature.

The existing stability conditions can be classified into two types: delay-dependent stability conditions and delay-independent stability conditions. Generally speaking, delay-dependent stability conditions are less conservative than delay-independent ones, when the size of time delay is small. However, few results have been investigated to the problem of stability for uncertain distributed time-delay systems, which exists room for further study.

In this paper, the problem of delay-dependent stability criterion for uncertain distributed time-delay systems is considered. By choosing suitable Lyapunov-Krasovskii functional and using free weighting matrices, new delay-dependent stability criterion is derived to guarantee the stability of the uncertain distributed time-delay systems. The obtained criterion is in terms of LMI, which can be solved efficiently by using the Matlab Toolbox.

\section{Problem statement}

Consider the following uncertain distributed system with time-varying delay

$$
\left\{\begin{array}{l}
\dot{x}(t)=(A+\Delta A(t)) x(t)+(B+\Delta B(t)) x(t-h(t))+(C+\Delta C(t)) \int_{t-h(t)}^{t} x(s) d s \\
x(t)=\varphi(t), s \in[-h, 0]
\end{array}\right.
$$

where $x(t) \in R^{n}$ is the state vector, $A, B$ and $C$ are constant matrices with appropriate dimensions. $\triangle A, \Delta B$ and $\triangle C$ denote the parameter uncertainties satisfying the following conditions:

$$
\left[\begin{array}{ll}
\Delta A & \Delta B
\end{array} \mathrm{C}\right]=D F(t)\left[\begin{array}{lll}
E_{1} & E_{2} & E_{3}
\end{array}\right]
$$


where $D, E_{1}, E_{2}$ and $E_{3}$ are constant matrices with appropriate dimensions and $F(t)$ is an unknown time-vayring matrix satisfying $F^{T}(t) \cdot F(t) \leq I . \varphi(t)$ is the initial function of the system. The time delay, $h(t)$, is a time-varying continuous function satisfying

$$
0 \leq h(t) \leq h, \dot{h}(t) \leq d, \forall t \geq 0
$$

where $h$ and $d$ are constants.

In the following, we will develop stability criterion for the system described by (1). The following lemma is useful in driving the criterion.

Lemma 1(Schur complement) Given constant symmetric matrices $\Sigma_{1}, \Sigma_{2}, \Sigma_{3}$ where $\quad \Sigma_{1}=\Sigma_{1}^{T}$ and $0<\Sigma_{2}=\Sigma_{2}^{T}$, then $\Sigma_{1}+\Sigma_{3}^{T} \Sigma_{2}^{-1} \Sigma_{3}<0$ if and only if

$$
\left[\begin{array}{cc}
\Sigma_{1} & \Sigma_{3}^{T} \\
\Sigma_{3} & -\Sigma_{2}
\end{array}\right]<0 \text { or }\left[\begin{array}{cc}
-\Sigma_{2} & \Sigma_{3} \\
\Sigma_{3}^{T} & \Sigma_{1}
\end{array}\right]<0
$$

Lemma 2([2]) For any positive definite matrix $M>0$, scalar $\gamma>0$, and vector function $x:[0, \gamma] \rightarrow R^{n}$, such that the integrations concerned are well defined, the following inequality holds:

$$
\left(\int_{0}^{\gamma} x(s) d s\right)^{T} M\left(\int_{0}^{\gamma} x(s) d s\right) \leq \gamma \int_{0}^{\gamma} x^{T}(s) M x(s) d s
$$

Lemma 3([4]) Given appropriately dimensioned matrices $\psi, H, G$ with $\psi=\psi^{T}$, then $\psi+H F(t) G+G^{T} F^{T}(t) H^{T}<0$ holds for all $F(t)$ satisfying $F^{T}(t) F(t) \leq I$, if and only if for some $\varepsilon>0, \psi+\varepsilon H H^{T}+\varepsilon^{-1} G^{T} G<0$.

\section{Main result}

In this section, we will investigate the stability condition for system (1).

Theorem 1 Given scalars $h \geq 0$ and $d>0$, if there exist symmetric positive definite matrices $P, Q, Z_{1}, Z_{2}$, any matrix $M, N, H$ with appropriate dimensions, and a scalar $\varepsilon>0$ such that the following LMI holds:

where

$$
\Psi=\left[\begin{array}{ccccc}
\Psi_{11} & \Psi_{12} & M C+\varepsilon E_{1}^{T} E_{3} & -M+P+A^{T} H^{T} & M D \\
* & \psi_{22} & N C+\varepsilon E_{2}^{T} E_{3} & -N+C H^{T} & N D \\
* & * & -\frac{Z_{1}}{h}+\varepsilon E_{3}^{T} E_{3} & C^{T} H^{T} & 0 \\
* & * & * & -H+H^{T}+h Z_{2} & H D \\
* & * & * & * & -\varepsilon I
\end{array}\right]<0
$$

$$
\begin{gathered}
\Psi_{11}=Q+h Z_{1}+M A+A^{T} M^{T}-\frac{Z_{2}}{h}+\varepsilon E_{1}^{T} E_{1}, \\
\Psi_{12}=\frac{Z_{2}}{h}+M B+A^{T} N^{T}+\varepsilon E_{1}^{T} E_{2}, \\
\Psi_{22}=-(1-d) Q+N B+B^{T} N^{T}-\frac{Z_{2}}{h}+\varepsilon E_{2}^{T} E_{2} .
\end{gathered}
$$

Proof. Construct a Lyapunov-Krasovskii functional candidate as

$$
V(t)=x^{T}(t) P x(t)+\int_{t-h(t)}^{t} x^{T}(s) Q x(s) d s+\int_{-h}^{0} \int_{t+\theta}^{t} x^{T}(s) Z_{1} x(s) d s d \theta+\int_{-h}^{0} \int_{t+\theta}^{t} \dot{x}^{T}(s) Z_{2} \dot{x}(s) d s d \theta
$$

where $P>0, Q>0, Z_{1}>0, Z_{2}>0$ are matrices to be determined. Taking the time derivative of $V(t)$ along the state trajectory (1) yields

$$
\begin{gathered}
\dot{V}(t)=2 x^{T}(t) P \dot{x}(t)+x^{T}(t) Q x(t)-(1-\dot{h}(t)) x^{T}(t-h(t)) Q x(t-h(t))+h x^{T}(t) Z_{1} x(t) \\
+h \dot{x}^{T}(t) Z_{2} \dot{x}(t)-\int_{t-h}^{t} x^{T}(s) Z_{1} x(s) d s-\int_{t-h}^{t} \dot{x}^{T}(s) Z_{2} \dot{x}(s) d s
\end{gathered}
$$




$$
\begin{aligned}
& \leq 2 x^{T}(t) P \dot{x}(t)+x^{T}(t) Q x(t)-(1-d) x^{T}(t-h(t)) Q x(t-h(t))+h x^{T}(t) Z_{1} x(t) \\
+ & h \dot{x}^{T}(t) Z_{2} \dot{x}(t)-\int_{t-h(t)}^{t} x^{T}(s) Z_{1} x(s) d s-\int_{t-h(t)}^{t} \dot{x}^{T}(s) Z_{2} \dot{x}(s) d s
\end{aligned}
$$

Applying lemma 2 to the last two integral terms in (2) and after simple manipulations, we have

$$
\begin{gathered}
-\int_{t-h(t)}^{t} x^{T}(s) Z_{1} x(s) d s \leq-\frac{1}{h(t)}\left(\int_{t-h(t)}^{t} x(s) d s\right)^{T} Z_{1}\left(\int_{t-h(t))}^{t} x(s) d s\right) \\
\leq-\frac{1}{h}\left(\int_{t-h(t)}^{t} x(s) d s\right)^{T} Z_{1}\left(\int_{t-h(t))}^{t} x(s) d s\right) \\
-\int_{t-h(t)}^{t} \dot{x}^{T}(s) Z_{2} \dot{x}(s) d s \leq-\frac{1}{h(}\left(\int_{t-h(t)}^{t} \dot{x}(s) d s\right)^{T} Z_{2}\left(\int_{t-h(t))}^{t} \dot{x}(s) d s\right) \\
=-\frac{1}{h}\left[x^{T}(t)-x^{T}(t-h(t))\right] Z_{2}\left[x^{T}(t)-x^{T}(t-h(t))\right]
\end{gathered}
$$

For any matrices $M, N, H$ with appropriate dimensions, the following equation is true

$$
\begin{aligned}
& 2\left[x^{T}(t) M+x^{T}(t-h(t)) N+\dot{x}^{T}(t) H\right] \cdot[(A+\Delta A(t)) x(t)+(B+\Delta B(t)) x(t-h(t)) \\
& \left.+(C+\Delta C(t)) \int_{t-h(t)}^{t} x(s) d s-\dot{x}(t)\right]=0
\end{aligned}
$$

Hence, according to (2)-(5), we can obtain

where

$$
V(t) \leq \xi^{T}(t) \Pi \xi(t)
$$

$$
\begin{gathered}
\xi(t)=\left[x^{T}(t) x^{T}(t-h(t)) \int_{t-h(t)}^{t} x^{T}(s) d s \dot{x}^{T}(t)\right]^{T}, \\
\Pi=\left[\begin{array}{cccc}
\Pi_{11} & \Pi_{12} & M(C+\Delta C(t)) & -M+P+(A+\Delta A(t))^{T} H^{T} \\
* & \Pi_{22} & N(C+\Delta C(t)) & -N+(B+\Delta B(t))^{T} H^{T} \\
* & * & -\frac{Z_{1}}{h} & (C+\Delta C(t))^{T} H^{T} \\
* & * & * & -H-H^{T}+h Z_{2}
\end{array}\right], \\
\Pi_{11}=Q+h Z_{1}+M(A+\Delta A(t))+(A+\Delta A(t))^{T} M^{T}-\frac{Z_{2}}{h}, \\
\Pi_{22}=-(1-d) Q+N(B+\Delta B(t))+(B+\Delta B(t))^{T} N^{T}-\frac{Z_{2}}{h}
\end{gathered}
$$

Notice that by applying Schur Complement and lemma $3, \Pi<0$ is equivalent to

$$
\begin{gathered}
{\left[\begin{array}{cccccc}
Q+h Z_{1}+M A+A^{T} M^{T}-\frac{Z_{2}}{h} & \multicolumn{2}{c}{\frac{Z_{2}}{h}+M B+A^{T} N^{T}} & M C & -M+P+A^{T} H^{T} & M D \\
* & & -(1-d) Q+N B+B^{T} N^{T}-\frac{Z_{2}}{h} & N C & -N+C H^{T} & N D \\
* & * & & -\frac{Z_{1}}{h} & C^{T} H^{T} & 0 \\
* & * & * & -H+H^{T}+h Z_{2} & H D \\
* & & * & * & * & -\varepsilon I
\end{array}\right]} \\
+\varepsilon\left[\begin{array}{llllllll}
E_{1} & E_{2} & E_{3} & 0 & 0
\end{array}\right]^{T}\left[\begin{array}{lllllll}
E_{1} & E_{2} & E_{3} & 0 & 0
\end{array}\right]<0
\end{gathered}
$$

By simple manipulations, the inequality (6) is equivalent to $\Psi<0$, which completes the proof. 


\section{Conclusions}

This paper is concerned with robust stability analysis for distributed time-delay systems with norm-bounded time-varying parameter uncertainties. Applying Lyapunov-Krasovskii functional and free weighting matrices, a new robust stability analysis criterion has been given in terms of LMI, which is dependent on the size of the time delay and can be easily verified by Matlab LMI toolbox.

\section{Acknowledgments}

This work is supported by the Natural Science Foundation of Hubei Province, China (No. 2011CDC028), the Key Project of Hubei Provincial Department of Education (No. D20122701 and No. D20102902), the Ph.D. Fund of Huanggang Normal University (No. 2011CD229 and to Cheng Wang).

\section{References}

[1] E. Fridman and U. Shaked, Delay-dependent stability and $H_{\infty}$ control: constant and time-varying delays, International Journal of Control 76 (2003) 48-60.

[2] C. Wang and Y. Shen, Improved delay-dependent robust stability criteria for uncertain time-delay systems, Applied Mathematics and Computation 218 (2011) 2880-2888.

[3] M.N.A. Parlakli, Robust stability of uncertain time-varying state-delayed systems, IEE Proceedings-Control Theory and Application 153 (2006) 469-477.

[4] P. Balasubramaniam, R. Chandran, Delay decomposition approach to stability analysis for uncertain fuzzy Hopfield neural networks with time-varying delay, Commum Nonlinear Sci Numer Simulat 16 (2011) 2098-2108.

[5] T. Li, L. Guo, Y. Zhang, Delay-range-dependent robust stability and stability for uncertain systems with time-varying delay, International Journal of Robust and Nonlinear Control 18 (2008) 1372-1387. 